ORIGINAL ARTICLE

\title{
Psychological predictors of injury among elite athletes
}

\author{
S A Galambos, P C Terry, G M Moyle, S A Locke
}

Br J Sports Med 2005;39:351-354. doi: 10.1136/bjsm.2005.018440

See end of article for authors' affiliations .......................

Correspondence to: Dr Galambos, Queensland Academy of Sport, PO Box 956, Sunnybank, QLD 4109, Australia; sandor. galambos@srq.qld.gov.au

Accepted 6 March 2005

\begin{abstract}
Objectives: To establish injury rates among a population of elite athletes, to provide normative data for psychological variables hypothesised to be predictive of sport injuries, and to establish relations between measures of mood, perceived life stress, and injury characteristics as a precursor to introducing a psychological intervention to ameliorate the injury problem.

Methods: As part of annual screening procedures, athletes at the Queensland Academy of Sport report medical and psychological status. Data from 845 screenings ( 433 female and 412 male athletes) were reviewed. Population specific tables of normative data were established for the Brunel mood scale and the perceived stress scale.

Results: About $67 \%$ of athletes were injured each year, and about $18 \%$ were injured at the time of screening. Fifty percent of variance in stress scores could be predicted from mood scores, especially for vigour, depression, and tension. Mood and stress scores collectively had significant utility in predicting injury characteristics. Injury status (current, healed, no injury) was correctly classified with $39 \%$ accuracy, and back pain with $48 \%$ accuracy. Among a subset of 233 uninjured athletes (1 16 female and 117 male), five mood dimensions (anger, confusion, fatigue, tension, depression) were significantly related to orthopaedic incidents over the preceding 12 months, with each mood dimension explaining 6-7\% of the variance. No sex differences in these relations were found.

Conclusions: The findings support suggestions that psychological measures have utility in predicting athletic injury, although the relatively modest explained variance highlights the need to also include underlying physiological indicators of allostatic load, such as stress hormones, in predictive models.
\end{abstract}

$\mathrm{R}$ isk of injury in sport and psychological factors that influence injury rehabilitation are well documented. ${ }^{1-3}$ Knowledge of psychological predictors of injury in elite sport is far from comprehensive, and findings to date have been somewhat contradictory. A stress and sport injury model originally proposed by Andersen and Williams ${ }^{45}$ was recently extended by Petrie and Perna. ${ }^{6}$ The model posits that the effects of psychological risk factors on injuries and other adverse sport related health outcomes are mediated by the cumulative effect of acute and/or chronic physiological stress responses. A substantial body of research consistently supports the relations between injury and psychometric indices of stressful life events in specific athletic populations, although the directly explained variances are often modest. ${ }^{5}$

The relation between psychometric and physiological stress level indicators and injury outcomes has not been formally investigated in elite sports, although a recent study on 40 collegiate rowers who received cognitive-behavioural stress management training found that it halved injury/illness incidence, with an even greater reduction in lost training time and corresponding negative affect and baseline cortisol concentration reductions. ${ }^{7}$ Despite the lack of formal investigations, a positive relation between cortisol as a cumulative physiological stress indicator, perceived stress, and negative affect is well supported by recovery research in sport sciences and by clinically directed psychoneuroendocrinological, occupational health, and psychology oriented investigations. ${ }^{8-11}$ Limited support also exists for a direct positive relation between injury and the affective variables of tension, anxiety, hostility, and outward directed anger..$^{12}$

The Queensland Academy of Sport (QAS) supports nearly 700 athletes in 20 sports. Given the cost of injuries, in terms of time out of sport with consequent detraining, the expense of rehabilitation, and adverse social and economic effects, ${ }^{3}$ comprehensive injury management and prevention has become a priority for the QAS. Considering the potential for developing cost effective, preventive programmes, knowledge gained by examination of psychological predictors of injury may also prove beneficial for the broader sports medicine community.

In light of the above, the primary objective of this study was to examine the reproducibility of the relation between sport related orthopaedic incidents (including traumatic injuries), lost training time, and the psychological variables of life stress and negative mood indicators in a mixed, elite athlete population-scholarship athletes at QAS. As a prerequisite for future prospective research, the secondary objective of the study was to see whether the standard psychometric measures of mood and perceived stress used by QAS in its screening procedures consistently relate to one another and to injury variables. Related to the latter objective, tables of normative data for the mood and life stress measurement scales were developed as a baseline for ongoing research. The present research was facilitated by examination of archival data obtained by methods already in place at the QAS as part of their medical screening processes.

\section{METHODS}

QAS athletes complete a 374 item health screening questionnaire annually to record, among other things, full medical history, incidents during the preceding 12 months, training characteristics, and current medical and psychological status. Data from 845 questionnaires (433 female and 412 male athletes) collected between 2002 and 2004 were used in the present research. Athletes ranged from 11 to 41 years of age (mean (SD) 18.8 (4.6)). Sports represented were athletics, basketball, beach volleyball, canoeing, cricket, cycling, golf, gymnastics, hockey, netball, rugby league, rugby union, soccer, softball, swimming, tennis, triathlon, and water polo. Norms for psychometric measures were based on the whole sample. A subset of 233 uninjured athletes (116 female and 117 male) was used in some 
Table 1 Elite athlete norms for the Brunel Mood Scale ( $n=845$ )

\begin{tabular}{|c|c|c|c|c|c|c|}
\hline \multirow[b]{2}{*}{ Raw score } & \multicolumn{6}{|l|}{ T score } \\
\hline & Anger & Confusion & Depression & Fatigue & Tension & Vigour \\
\hline 0 & 39 & 41 & 43 & 33 & 39 & 18 \\
\hline 1 & 43 & 45 & 47 & 36 & 42 & 21 \\
\hline 2 & 46 & 48 & 51 & 39 & 46 & 25 \\
\hline 3 & 50 & 52 & 55 & 42 & 50 & 28 \\
\hline 4 & 54 & 56 & 58 & 44 & 54 & 31 \\
\hline 5 & 58 & 60 & 62 & 47 & 57 & 35 \\
\hline 6 & 61 & 63 & 66 & 50 & 61 & 38 \\
\hline 7 & 65 & 67 & 70 & 52 & 65 & 41 \\
\hline 8 & 69 & 71 & 74 & 55 & 69 & 45 \\
\hline 9 & 73 & 74 & 78 & 58 & 73 & 48 \\
\hline 10 & 76 & 78 & 82 & 61 & 76 & 51 \\
\hline 11 & 80 & 82 & 86 & 63 & 80 & 54 \\
\hline 12 & 84 & 86 & 90 & 66 & 84 & 58 \\
\hline 13 & 88 & 89 & 94 & 69 & 88 & 61 \\
\hline 14 & 91 & 93 & 98 & 72 & 91 & 64 \\
\hline 15 & 95 & 97 & 102 & 74 & 95 & 68 \\
\hline 16 & 99 & 100 & 106 & 77 & 99 & 71 \\
\hline
\end{tabular}

analyses to eliminate effects of current injury, which tends to inflate psychometric scores. Athletes with ongoing consequences of prior injuries requiring orthopaedic or orthotic devices were also excluded. Athletes in this subset ranged from 12 to 38 years of age (mean $18.6(4.4)$ ).

Injury was operationalised as all medically attended, sport related somatic damage. ${ }^{14}$ Orthopaedic history-that is, never/within one year/more than 12 months ago-was recorded for 12 body regions and included common sport related conditions such as back pain and bursitis in addition to injuries. Injury status - that is, healed/not healed-at the time of screening was also recorded. Training and competition days lost to injury were recorded - that is, nil, 1-7 days, 8-14 days, and >14 days-and summed into loss units, where 1-7 days equals one unit, 8-14 days equals two units, and so on. ${ }^{15}$

The psychology section, added in 2002, includes history of diagnoses (for example, anxiety disorder, eating disorders), identification of disordered eating behaviours (for example, bingeing, self induced vomiting), and two standardised measures: the Brunel Mood Scale ${ }^{1617}$ and the Perceived Stress Scale-10. ${ }^{18}{ }^{19}$ The Brunel Mood Scale is a 24 item, self report inventory of six subscales (anger, confusion, depression, fatigue, tension, vigour). The depression subscale is an indicator of depressed mood, not clinical depression. Respondents indicate whether they have felt-for example, angry, energetic, nervous, or unhappy-on a five point Likert scale $(0=$ not at all, $1=$ a little, $2=$ moderately, $3=$ quite a bit, $4=$ extremely). Subscale scores range from 0 to 16 . The standard response time frame is "How you feel right now", although for the purposes of screening "How you have felt during the past month including today" was used. The Brunel Mood Scale has been shown to be valid and reliable among both adolescent ${ }^{16}$ and adult ${ }^{17}$ athlete populations.

The Perceived Stress Scale-10 is a 10 item, self report inventory that assesses the degree to which situations in an individual's life are appraised as stressful. Respondents indicate how often they have felt or thought a certain way on a five point Likert scale $(0=$ never, $1=$ almost never, 2 $=$ sometimes, $3=$ fairly often, $4=$ very often). The standard response time frame is "In the last month" - for example, "how often have you felt that you were unable to control the important things in your life?" Scores range from 0 to 40 with no specific categories or cut offs suggested by the authors. ${ }^{19}$ Ethical approval for this investigation was granted by the QAS Research Committee.

Table 3 Mood and life stress scores by injury status

\begin{tabular}{llll}
\hline Source & $\begin{array}{l}\text { No injury } \\
\text { (n= 269) }\end{array}$ & $\begin{array}{l}\text { Healed injury } \\
\text { (n= 389) }\end{array}$ & $\begin{array}{l}\text { Current injury } \\
\text { (n= 144) }\end{array}$ \\
\hline Anger & $2.50(2.46)$ & $2.92(2.69)$ & $3.25(2.89) \dagger$ \\
Confusion & $2.06(2.61)$ & $2.40(2.61)$ & $2.81(2.85) \dagger$ \\
Depression & $1.46(2.36)$ & $1.73(2.34)$ & $2.40(2.90) \dagger$ \\
Fatigue & $5.61(3.61)$ & $6.06(3.64)$ & $6.94(3.93) \dagger$ \\
Tension & $2.69(2.62)$ & $3.11(2.83)$ & $3.42(2.90) \dagger$ \\
Vigour & $9.44(3.14)$ & $9.89(2.98)$ & $9.47(3.13)$ \\
Life stress & $10.86(5.74)$ & $12.14(5.62)$ & $13.13(6.34) \dagger$ \\
\hline
\end{tabular}

Values are mean (SD). Wilks $=0.96, p=0.003$

†Significantly different $(p<0.01)$ from no injury.

\begin{tabular}{|c|c|c|c|c|c|c|c|}
\hline Raw score & T score & Raw score & T score & Raw score & T score & Raw score & T score \\
\hline 0 & 29 & & & & & & \\
\hline 1 & 31 & 11 & 48 & 21 & 65 & 31 & 82 \\
\hline 2 & 33 & 12 & 50 & 22 & 67 & 32 & 84 \\
\hline 3 & 34 & 13 & 52 & 23 & 69 & 33 & 86 \\
\hline 4 & 36 & 14 & 53 & 24 & 70 & 34 & 87 \\
\hline 5 & 38 & 15 & 55 & 25 & 72 & 35 & 89 \\
\hline 6 & 40 & 16 & 57 & 26 & 74 & 36 & 91 \\
\hline 7 & 41 & 17 & 58 & 27 & 75 & 37 & 93 \\
\hline 8 & 43 & 18 & 60 & 28 & 77 & 38 & 94 \\
\hline 9 & 45 & 19 & 62 & 29 & 79 & 39 & 96 \\
\hline 10 & 46 & 20 & 64 & 30 & 81 & 40 & 98 \\
\hline
\end{tabular}




\section{What is already known on this topic}

Psychometric mood and perceived stress indicators provide useful information in terms of explaining injury risk in athletes, although variances explained by these indicators alone are usually modest.

\section{RESULTS}

\section{Mood and life stress norms}

Initial sex comparisons showed no meaningful differences between the mood and life stress reports of male and female athletes, and hence their data were combined for further analyses. Tables of normative data for the mood and stress scales specific to the present population of elite athletes were produced (tables 1 and 2). Mean scores for several of the mood scales differed significantly from existing norms for adult athletes. ${ }^{16}$ In the present sample, compared with existing norms, mean scores for anger, confusion, depression, fatigue, tension, and vigour fell at the 65th, 52nd, 57th, 61st, 46th, and 55th centiles respectively.

These differences can be explained partly by use of the "past month" response time frame compared with "right now" for the original norms, which has been shown previously to generally inflate scores on mood scales. ${ }^{20}$ However, the lower tension scores run counter to this trend, and the high mean scores for anger and fatigue, which are respectively 1.5 and 1 standard deviations above the existing norms, are highly suggestive that the current population of athletes typically report moods that are uncharacteristic of general athletic populations, thereby providing justification for a population specific table of normative data.

\section{Mood and stress relations}

Multiple regression analysis showed that mood scores collectively predicted $50 \%$ of the variance in life stress $\left(F_{6,817}=138.96, \mathrm{p}<0.001\right)$. As expected, high life stress was predicted by high anger $(t=3.41, \mathrm{p}=0.001)$, confusion $(t=3.09, \mathrm{p}=0.002)$, depression $(t=6.82$, $\mathrm{p}<0.001)$, fatigue $(t=2.92, \mathrm{p}=0.004)$, and tension $(t=$ $6.76, \mathrm{p}<0.001)$ and low vigour $(t=-8.52, \mathrm{p}<0.001)$.

\section{Injury status}

The overall incidence of athletes reporting at least one injury in the previous 12 months was $67.1 \%$, with $17.3 \%$ of athletes reporting two or more injuries. At the time of screening, $18.2 \%$ of athletes were injured. Multivariate analysis of variance showed significantly elevated life stress and significant mood disturbance on all mood scales except vigour among athletes with a current injury compared with those who had not been injured in the preceding 12 months
What this study adds

Further support is provided for including stress and mood indices in explanatory models of sport injury by using a relatively large and diverse population of high level athletes. The need for concurrent investigations of psychological and physiological injury risk indicators is emphasised, as the modest predictive utility of psychometric indicators is in contrast with a substantial injury prevention potential reported by a few recent psychological intervention studies.

(table 3). Discriminant function analysis showed that athletes could be correctly classified into injury groups-that is, current, healed, no injury-from mood and life stress scores with $39 \%$ accuracy, compared with $33 \%$ by chance alone. Using back pain as a more specific exemplar, the overall incidence was $34 \%$, with $21 \%$ of athletes reporting current or recent $(<12$ months) back pain and 13\% reporting previous back pain (>12 months). The same pattern of significantly increased life stress and significant mood disturbance among those with current back pain was evident. Athletes could be correctly classified into back pain groupsthat is, current, previous, never-from mood and life stress scores with $48 \%$ accuracy.

\section{Uninjured athletes}

To avoid the impact of current injury on psychological status, relations between mood, perceived stress, injury, and corresponding time lost were investigated among 233 athletes who had either remained uninjured during the preceding 12 months or were uninjured at the time of screening (table 4). The number of orthopaedic incidents in this group ranged from 0 to 12 with a mean of 1.2. Time lost through injury ranged from 0 to 105 days with a mean of about one week.

The small but significant correlations between negative mood indicators (anger, confusion, depression and fatigue), perceived stress, injuries, and time lost are consistent with previous findings. ${ }^{5}$ Collectively, mood and stress scores explained $9.6 \%$ of variance in number of injury incidents $\left(F_{7,225}=3.43, \mathrm{p}=0.002\right)$ and $9.9 \%$ of variance in time lost to injury $\left(F_{7,225}=3.43, \mathrm{p}=0.001\right)$. High depression scores and low vigour scores were significant predictors of time lost.

\section{DISCUSSION}

These results illustrate the scale of the injury problem in elite sport and the need for interventions to reduce it. The population specific norms generated in this study provide baseline indicators that may be useful in future research, especially if ongoing analysis of completed screenings refines their utility.

Table 4 Intercorrelations among mood, stress, injury, time loss, and training hours for 233 uninjured athletes

\begin{tabular}{llllllllll}
\hline Source & $\mathbf{A}^{*}$ & $\mathbf{C}$ & $\mathbf{D}$ & $\mathbf{F}$ & $\mathbf{T}$ & $\mathbf{V}$ & $\mathbf{S}$ & $\mathbf{I}$ & $\mathbf{L}$ \\
\hline Confusion (C) & $0.76 \dagger$ & & & & & & & & \\
Depression (D) & $0.75 \dagger$ & $0.73 \dagger$ & & & & & & & \\
Fatigue (F) & $0.58 \dagger$ & $0.48 \dagger$ & $0.48 \dagger$ & & & & & & \\
Tension (T) & $0.66 \dagger$ & $0.67 \dagger$ & $0.69 \dagger$ & $0.45 \dagger$ & & & & & \\
Vigour (V) & 0.06 & 0.06 & -0.02 & 0.13 & $0.12 \ddagger$ & & & & \\
Stress (S) & $0.43 \dagger$ & $0.50 \dagger$ & $0.42 \dagger$ & $0.46 \dagger$ & $0.51 \dagger$ & $0.37 \dagger$ & & & \\
Injury (I) & $0.27 \dagger$ & $0.26 \dagger$ & $0.23 \dagger$ & $0.26 \dagger$ & $0.24 \dagger$ & 0.06 & $0.15 \ddagger$ & & \\
Time lost (L) & 0.12 & 0.10 & $0.20 \ddagger$ & $0.14 \ddagger$ & 0.22 & $0.18 \ddagger$ & $0.19 \ddagger$ & $0.30 \dagger$ & \\
\hline${ }^{*} \mathrm{~A}=$ anger. & & & & & & & & & \\
$t p<0.01 ; \neq \mathrm{p}<0.05$. & & & & & & & & & \\
\hline
\end{tabular}


The findings show that measures of mood and perceived life stress predicted injury related variables, although the explained variance was modest, which is consistent with previous studies. It appears unlikely that substantial practical advancement in prevention is achievable with exclusive reliance on psychological factors. High level athletes depend on rapid proactive adjustments to changing physical demands, a process that requires indices of demand relative to capacity. Self report measures have a role to play in this process but may need to be augmented by cumulative physiological indicators of injury risk, such as free cortisol concentration. Although rare, where such a strategy has been reported it has shown significant potential for positively influencing both psychological and physiological risk indicators and corresponding injury incidences and lost training time. $^{72122}$

Contemporary models to explain sport injury emphasise the cumulative effects of physical and psychological stressors that deplete perceptual and sensory motor reserves which might help an athlete to avoid injury. ${ }^{4} 6$ In behavioural medicine, the term allostasis is used to describe the deviation of physiological parameters, such as sympathoadrenal and hypothalamic-pituitary-adrenal mediator and hormone productions, from homoeostatic levels. ${ }^{10}$ Hyperactivity of the allostatic systems is known to be related to cardiovascular, immunological, and neuropsychological-behavioural disturbances and illnesses both acutely and cumulatively. ${ }^{10}{ }^{23}$ As high allostatic load is inevitable in most high level sports, it is reasonable to assume that the capacity to respond appropriately to acute, superimposed physical and/or psychological stressors that add to the sport related allostatic pre-load will be limited. Furthermore, as psychological stressor effects are mediated through the same pathways as physical stressors, ${ }^{24}$ athletes subjected to recent stressful life events have a higher compound allostatic load and even more diminished threshold for mounting situational appropriate emergency responses. ${ }^{925}$

An athlete in an overreaching phase of training or intense competition would appear to be particularly vulnerable. As the potential to eliminate physical stressors is limited in sport, a potential avenue for decreasing injury rates is to control or eliminate unnecessary psychological stressors, thereby increasing reserves for responding in an emergency. There is a need to conduct further investigations of the injury reducing capacity of interventions designed to lower allostatic load.

\section{Authors' affiliations}

S A Galambos, P C Terry, G M Moyle, S A Locke, Queensland Academy of Sport, Centre of Excellence for Applied Sports Science Research, Brisbane, Australia

Competing interests: none declared

\section{REFERENCES}

1 Brewer BW. Developmental differences in psychological aspects of sportinjury rehabilitation. J Athl Train 2003;38:152-3.

2 Johnson U. A three-year follow-up of long-term injured competitive athletes: influence of psychological risk factors on rehabilitation. Journal of Sport Rehabilitation 1997;6:256-71.

3 Van Mechelen W, Hlobil H, Kemper HCG. Incidence, severity, aetiology and prevention of sports injuries: a review of concepts. Sports Med 1992; 14:82-99

4 Andersen MB, Williams JM. A model of stress and athletic injury: prediction and prevention. Journal of Sport and Exercise Psychology 1988;10:294-306.
5 Williams JM. Psychology of injury risk and prevention. In: Singer R, Hausenblas H, Janelle C, eds. Handbook of sport psychology. New York: John Wiley \& Sons, 2001:766-86.

6 Petrie TA, Perna F. Psychology of injury: theory, research and practice. In: Morris T, Summers J, eds. Sport psychology:theory, applications and issues.2nd ed. Milton, QLD: John Willey \& Sons, 2004:547-71.

7 Perna FM, Antoni MH, Baum A, et al. Cognitive behavioral stress management effects on injury and illness among competitive athletes: a randomized clinical trial. Ann Behav Med 2003:25:66-73.

8 Frankenhaeuser S. A psychobiological framework for human stress and coping. In: Appley MH, Trumbull R, eds. Dynamics of stress: physiological, psychological, and social perspectives. New York: Plenum Press, 1986:101-16.

9 Davis H, Botterill C, MacNeill K. Mood and self-regulation changes in underrecovery: an intervention model. In: Kellmann M, eds. Enhancing recovery: preventing underperformance in athletes. Champaign, IL: Human Kinetics, 2002:161-79.

10 McEwen BS. Stress and neuroendocrine function. In: Wolkowitz OM, Rothschild AJ, eds. Psychoneuroendocrinology: the scientific basis of clinical practice, Arlington, VA:American Psychiatric Publishing, 2003:513-46.

11 Fogarty GJ, Machin MA, Albion MJ, et al. Predicting occupational strain and job satisfaction: the role of stress, coping, personality and affective variables. $J$ Vocat Behav 1999;54:429-52.

12 Thompson NJ, Morris RD. Predicting injury risk in adolescent football players: the importance of psychological variables. J Pediatr Psychol 1994; 19:415-29.

13 Lavallee L, Flint F. Relationship of stress, competitive anxiety, mood state and social support to athletic injury. J Athl Train 1996;31:296-9.

14 Finch C. An overview of some definitional issues for sports injury surveillance. Sports Med 1997;24:157-63.

15 Petrie TA, Falkstein DL. Methodological, measurement, and statistical issues in research on sport injury prediction. J Appl Sport Psychol 1998;10:26-45.

16 Terry PC, Lane AM, Lane HJ, et al. Development and validation of a mood measure for adolescents. J Sports Sci 1999;17:861-72.

17 Terry PC, Lane AM, Fogarty GJ. Construct validity of the POMS-A for use with adults. Psychology of Sport and Exercise 2003;4:125-39.

18 Cohen S, Kamarck T, Mermelstein R. A global measure of perceived stress. $J$ Health Soc Behav 1983;24:386-96.

19 Cohen S, Williamson GM. Perceived stress in a probability sample of the United States. In: Spacapan S, Oskamp S, eds. The social psychology of health: Claremont symposium on applied social psychology. Newbury Park, CA: Sage, 1988

20 Winkielman P, Knauper B, Schwarz N. Looking back at anger: reference periods change the interpretation of emotion frequency questions. J Pers Soc Psychol 1998;75:719-28.

21 Perna FM, Antoni MH, Kumar M, et al. Cognitive-behavioral intervention effects on mood and cortisol during athletic training. Ann Behav Med 1998;20:92-8

22 Kerr G, Goss J. The effects of a stress management program on injuries and stress levels. J Appl Sport Psychol 1996;8:109-17.

23 McEwen BS. Protective and damaging effects of stress mediators. N Engl J Med 1998;338:171-9.

24 Ganong WF. Review of medical physiology, 21 st ed. New York: Lange Medical Books/McGraw-Hill, 2003.

25 Steinacker JM, Lehmann M. Clinical findings and mechanisms of stress recovery in athletes. In: Kellmann $M$, ed. Enhancing recovery: preventing underperformance in athletes. Champaign, IL: Human Kinetics, 2002:103-18.
The relation between psychological variables and injury is an under-researched area. This study uses data collected as part of sport science screening processes. It explores relations between mood, stress, and injury. The results indicate that monitoring mood can provide useful information in terms of injury risk, supporting the value of monitoring mood in athletes.

A M Lane University of Wolverhampton, Walsall, UK; a.m.lane2@wlv.ac.uk 